Research Article

\title{
A Penetrating-Anchoring Mathematical Model for the Soft Asteroid Anchoring System
}

\author{
Zhijun Zhao ${ }^{D}{ }^{1}$ Shuang Wang, ${ }^{2}$ and Jingdong Zhao $\mathbb{D}^{3}$ \\ ${ }^{1}$ Beijing Key Laboratory of Intelligent Space Robotic Systems Technology and Applications, Beijing Institute of Spacecraft \\ System Engineering, Beijing 100092, China \\ ${ }^{2}$ Space Star Technology Co., Ltd, China \\ ${ }^{3}$ State Key Laboratory of Robotics and System, Harbin Institute of Technology, Harbin 150080, China \\ Correspondence should be addressed to Jingdong Zhao; zhaojingdong@hit.edu.cn
}

Received 11 January 2020; Revised 2 December 2020; Accepted 3 February 2021; Published 20 February 2021

Academic Editor: Jeremy Straub

Copyright (c) 2021 Zhijun Zhao et al. This is an open access article distributed under the Creative Commons Attribution License, which permits unrestricted use, distribution, and reproduction in any medium, provided the original work is properly cited.

\begin{abstract}
The asteroid landing mechanism is necessary to be anchored to avoid flowing away. At present, the study on the anchoring system is mainly focused on the mechanical design, but there are few researches on the penetrating or anchoring mathematical model, and the researches on combining two models with each other are even more lacking. In the paper, based on the characteristics of MohrCoulomb material, a penetrating mathematical model of the anchoring system is established. This penetrating mathematical model can be used to calculate the penetrating depth of the anchor body according to the penetrating speed and the medium properties. Secondly, an anchoring mathematical model is established, which shows the relationships among the anchoring force, medium properties, and penetrating depth. Finally, a penetrating-anchoring mathematical model is built with the penetrating depth as the link. The model establishes a relationship between the anchoring force and the initial penetrating conditions.
\end{abstract}

\section{Introduction}

Asteroid exploration has high scientific and economic values [1-3], such as finding evolution history of the planetary system, finding the origin of the solar system and the life, and exploring the asteroid resources. Moreover, asteroid exploration opens up a new field of deep space exploring $[4,5]$.

The landing mechanism must be fixed on the asteroid surface by the anchoring system to prevent flowing away due to the microgravity on the asteroid. The greater the anchoring force, the easier it is for the landing mechanism to be anchored on the asteroid surface. The anchoring force is closely related to the penetrating depth and the medium characteristics, and the penetrating depth is related to the mass of the anchor body, the shape of the anchor tip, the characteristics of the medium, and so on. Therefore, it is significant to analyze the penetrating-anchoring theory of the anchoring system on the asteroid surface. Penetrating analysis can predict penetrating depth of the anchoring system on the asteroid surface, and anchoring analysis can estimate the anchoring force that can be generated by the anchoring system at a certain penetration depth. Both of penetrating and anchoring analysis can guide the anchoring of the landing mechanism on the asteroid surface.

At present, the anchoring theory research on the asteroid landing is still in the starting state, and the relevant theoretical model has not been reported. Currently, anchoring theory is mainly about the pile foundation engineering. Ilamparuthi and Dickin summarized some anchoring models established by the pioneers [6] (Majer et al. (1955) established a vertical slip surface model. Downs et al. (1966), Murray et al. (1987), and Clemence et al. (1977) established an inverted truncated cone model. Balla (1961) and Meyerhof et al. (1968) established a curved slip surface model.). Sutherland studied the anchoring force of pile-based anchors in clay and analyzed various models [7]. Dickin studied the anchoring of anchors in sand media from 1988 to 1992 [8]. In 1994, Rao and Kumar studied the anchoring capacity of pile-based anchors and analyzed various models [9]. Ilamparuthi and Dickin studied the anchorage effects of different ends in the sand 
medium in 2001 [6]. Merifield et al. studied the anchoring of pile anchors in clay [10]. Wu et al. studied the anchoring of pile-based anchors in plaster-cement mixed media [11]. Shanker et al. studied the anchoring of the light rod [12]. Niroumand et al. studied the anchoring of the late expanded pile foundation in the sand medium [13]. Liu et al. studied the deformation of the medium during the drawing process of the anchor [14]. Consoli et al. studied the anchoring force of the pile body in cement [15]. The anchorage of the pile foundation is larger (about several meters in length), and the ground buried depth is deep (tens of meters). It is distinctly different from the anchoring system in the volume and penetration depth. Pile anchorage is different from asteroid anchoring to some extent, but it can be taken as guidance and reference for the study of the asteroid anchoring.

Currently, there are few researches on the anchoring model for the asteroid anchoring, let alone the penetratinganchoring model. However, the mathematical model describing the relationship between the penetrating and the anchoring is necessary in asteroid anchoring. In the paper, the vertical penetration equation of the anchoring system on the asteroid surface is derived. This equation can describe the mathematical relationship among the penetration speed, penetration depth, and the medium properties. Thus, this equation can predict the penetration depth of the anchor body if the medium property is known. Then, the anchoring model of the anchoring system is established. The model can be used to estimate the anchoring force generated by the anchoring system. Finally, the penetrating-anchoring model is established, which displays the relationship between the penetration velocity, the medium characteristics, and the anchoring force.

\section{Anchoring System}

The landing mechanism anchors on the asteroid via the anchoring system. The anchoring system contains penetrating unit, advancing unit, winding unit, damping unit, and so on. Its schematics and performances are shown separately in Figure 1. The mass of the system is about $1.5 \mathrm{~kg}$. The penetrating unit drafting a wire of about $2 \mathrm{~m}$ in length can penetrate the soft asteroid surface. Then, a wire connection is established between the landing mechanism and the asteroid.

Penetrating unit in the anchoring system is active and deformable, and its mechanical structure is shown in the top graph of Figure 2. The penetrating unit is mainly composed of anchor tip, anchor claw, barb, handspike, piston, pyrotechnics, anchor body, etc. As the penetrating unit penetrated the asteroid surface, the control system ignites the pyrotechnics and produces high-pressure gas. The gas pushes the handspike via the piston, inducing the splay of the claw and the barbs. The deployed penetrating unit is shown in the lower graph of Figure 2. In soft media, the claws and barbs are fully opened, and the open barbs increase the contact area between the anchor tip and the media, increasing the anchoring force. In hard media, the claws and barbs will generate irregular and plastic deformation when opening due to the great thrust generated by the pyrotechnic. This phe-

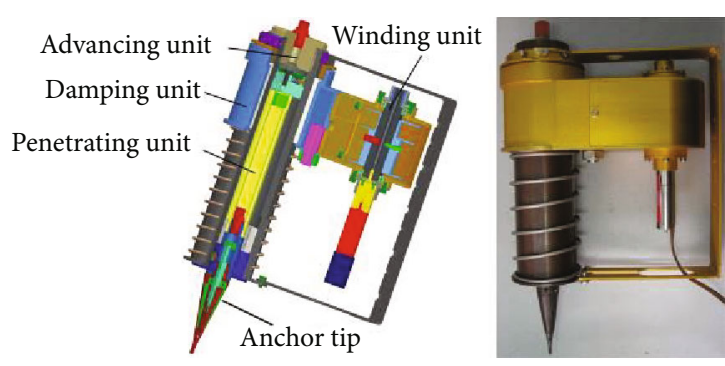

FIGURE 1: Schematics of anchoring system.

nomenon is similar to the effect of expansion bolt, and it can enhance the anchoring force.

The anchoring force is a key index of the anchoring system, directly related to its penetrating depth of the penetrating unit. However, this penetrating depth depends on the initial conditions such as the medium properties, the shape of the anchor tip, the mass, and penetrating velocity. Thus, it is necessary to establish the relationship between the anchoring force and the initial condition, which can provide theoretical foundation for reliable anchorage of the anchoring system.

\section{Penetrating Model}

3.1. Penetrating Resistance Force Analysis. The anchoring force is directly related to the penetrating depth. In order to establish the relationship between the anchoring force and the initial condition, it is firstly necessary to establish the relationship between the penetrating depth and the initial condition.

The forces on arbitrary shape anchor tip are shown in Figure 3 in penetrating.

Based on the cavity expansion model [16-18] and citing the deduction process of the paper [19], we can get the axial force on the anchor tip [18].

$$
\begin{gathered}
F_{z 1}=2 \pi \int_{0}^{r}\left(A \tau_{0}+B \rho_{0} V_{z}^{2} \frac{1}{1+\left[\varphi^{\prime}(r)\right]^{2}}\right) r d r+ \\
2 \pi \mu \int_{0}^{z}\left(A \tau_{0}+B \rho_{0} V_{z}^{2} \frac{\left[f^{\prime}(z)\right]^{2}}{1+\left[f^{\prime}(z)\right]^{2}}\right) f(z) d z 0 \leq z \leq p \\
F_{z 2}=2 \pi \int_{0}^{R}\left(A \tau_{0}+B \rho_{0} V_{z}^{2} \frac{1}{1+\left[\varphi^{\prime}(r)\right]^{2}}\right) r d r+ \\
2 \pi \mu \int_{0}^{p}\left(A \tau_{0}+B \rho_{0} V_{z}^{2} \frac{\left[f^{\prime}(z)\right]^{2}}{1+\left[f^{\prime}(z)\right]^{2}}\right) f(z) d z z>p,
\end{gathered}
$$




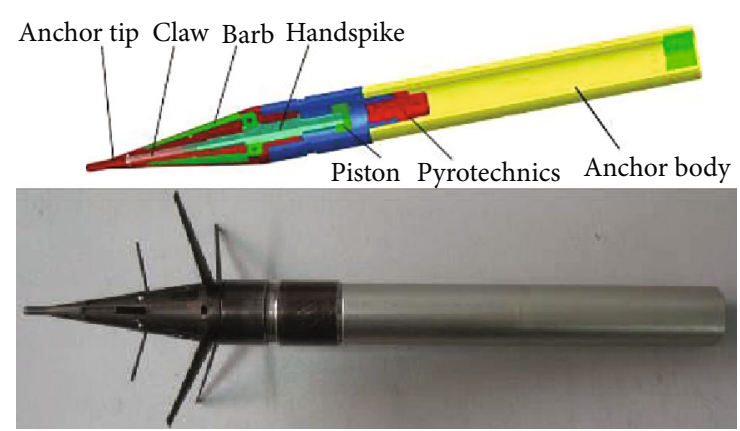

FIgURE 2: Schematic of the penetrating unit.

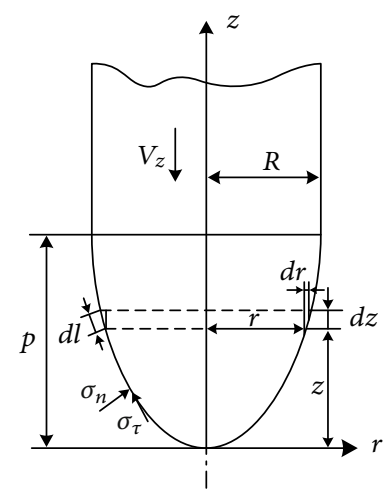

FiguRE 3: Force on arbitrary anchor tip.

where

$$
A=\frac{1}{\alpha}\left(\frac{1+\tau_{0} / 2 E}{\gamma}\right)^{2 \alpha}-\frac{1}{\lambda}
$$

$B=(3 /(1-\eta *)(1-2 \alpha)(2-\alpha))+\left(1 / \gamma^{2}\right)\left(1+\tau_{0} / 2 E / \gamma\right)^{2 \alpha}$ $\left\{\left(3 \tau_{0} / E\right)+\eta *\left(1-3 \tau_{0} / 2 E\right)^{2}-\gamma^{3}\left[2(1-\eta *)(2-\alpha)+3 \gamma^{3}\right] /(1\right.$ $\left.-\eta *)(1-2 \alpha)(2-\alpha)\left(1+\tau_{0} / 2 E\right)^{4}\right\}$.

$\alpha=3 \lambda / 3+2 \lambda$, where $\lambda=\tan \varphi$ and $\varphi$ is the internal friction angle.

$\eta *=1-\rho_{0} / \rho *$, where $\rho_{0}$ is the volume density before distortion and $\rho *$ is the volume density after distortion.

$$
\gamma=\frac{V}{c}=\left[\left(1+\frac{\tau_{0}}{2 E}\right)-(1-\eta *)\right]^{1 / 3},
$$

where $E$ is the Young's modulus and $\tau_{0}$ is the cohesion of the medium.

3.2. Penetrating Depth. The generatrix equation of the conic anchor tip is as follows:

$$
\frac{r}{z}=\frac{R}{p}=\tan \theta
$$

where $\theta$ is the semivertical angle of the anchor tip and $\theta=$ 9.46 .

\section{Obtaining}

$$
\begin{gathered}
\left\{\begin{array}{l}
r=f(z)=z \tan \theta, \\
z=\varphi(r)=r \cot \theta,
\end{array}\right. \\
\left\{\begin{array}{l}
f^{\prime}(z)=\tan \theta, \\
\varphi^{\prime}(r)=\cot \theta .
\end{array}\right.
\end{gathered}
$$
obtain

Integrating the above equation with equation (4) will

$$
\begin{gathered}
F_{z 1}=\pi r^{2}\left(A \tau_{0}+B \rho_{0} V_{z}^{2} \cdot \sin ^{2} \theta\right)+ \\
2 \pi \mu \int_{0}^{z}\left(A \tau_{0}+B \rho_{0} V_{z}^{2} \cdot \sin ^{2} \theta\right) z \tan \theta d z 0 \leq z \leq p \\
F_{z 2}=\pi R^{2}\left(A \tau_{0}+B \rho_{0} V_{z}^{2} \cdot \sin ^{2} \theta\right)+ \\
2 \pi \mu \int_{0}^{p}\left(A \tau_{0}+B \rho_{0} V_{z}^{2} \cdot \sin ^{2} \theta\right) z \tan \theta d z z>p .
\end{gathered}
$$

(1) When penetrating depth $0 \leq z \leq p$

To the conic anchor tip, according to equation (6),

$F_{z 1}=\pi r^{2}\left(A \tau_{0}+B \rho_{0} V_{z}^{2} \cdot \sin ^{2} \theta\right)(1+\mu \cot \theta) 0 \leq z \leq p$.

It can be written as follows:

$$
F_{z 1}=\pi r^{2} A \tau_{0}(1+\mu \cot \theta)+\pi r^{2} B \rho_{0} \sin ^{2} \theta(1+\mu \cot \theta) V_{z}^{2}
$$

Substituting $r=z \tan \theta$ into the equation above, it is obtained that

$$
F_{z 1}=\alpha_{1} z^{2}+\beta_{1} z^{2} V_{z}^{2}
$$

where

$$
\alpha_{1}=\pi \tan ^{2} \theta A \tau_{0}(1+\mu \cot \theta)
$$

$$
\beta_{1}=\pi \tan ^{2} \theta B \rho_{0} \sin ^{2} \theta(1+\mu \cot \theta) .
$$

According to Newton second law, we can get

$$
\begin{gathered}
m V_{z} \frac{d V_{z}}{d z}=-\left(\alpha_{1}+\beta_{1} V_{z}^{2}\right) z^{2} \\
\frac{m}{2} \frac{d V_{z}^{2}}{\left(\alpha_{1}+\beta_{1} V_{z}^{2}\right)}=-z^{2} d z
\end{gathered}
$$

Integrating

$$
-\frac{m}{2 \beta_{1}} \ln \left(\alpha_{1}+\beta_{1} V_{z}^{2}\right)+C_{1}=\frac{1}{3} z^{3} .
$$


When $t=0, V_{z}=V_{0}$, and $z=0$, we find

$$
C_{1}=\frac{m}{2 \beta_{1}} \ln \left(\alpha_{1}+\beta_{1} V_{0}^{2}\right)
$$

Substituting $C_{1}$ into equation (12), we can get

$$
z=\left[\frac{3 m}{2 \beta_{1}} \ln \frac{\left(\alpha_{1}+\beta_{1} V_{0}^{2}\right)}{\left(\alpha_{1}+\beta_{1} V_{z}^{2}\right)}\right]^{1 / 3}
$$

When $z=p$,

$$
V_{z p}=\sqrt{\left(\frac{\alpha_{1}}{\beta_{1}}+V_{0}^{2}\right) e^{-2 \beta_{1} p^{3} / 3 m}-\frac{\alpha_{1}}{\beta_{1}}} .
$$

When maximum penetrating depth $h_{1}<p$, setting $V_{z}=0$ , we can get

$$
h_{1}=\left[\frac{3 m}{2 \beta_{1}} \ln \left(1+\frac{\beta_{1}}{\alpha_{1}} V_{0}^{2}\right)\right]^{1 / 3} .
$$
get

By the way, according to the Newton second law, we can

$$
m \frac{d^{2} z}{d t^{2}}=-\left(\alpha_{1}+\beta_{1} V_{z}^{2}\right) z^{2}
$$

As $V_{z}=d z / d t$, the above equation can be written as

$$
m \frac{d^{2} z}{d t^{2}}=-\left[\alpha_{1}+\beta_{1}\left(\frac{d z}{d t}\right)^{2}\right] z^{2} .
$$

Integrating the above equation, we can get the relation between the depth $z$ and the time $t$. And then differentiating, we can get the relation between the acceleration and the time. In the paper, we are only interested in the relationship between the initial penetration velocity and the final penetration depth. Thus, the solution to the equation is not discussed in detail.

(2) When the penetrating depth $z>p$

When $z>p$, we can get the antiforce of the anchor tip by equation (6).

$F_{z 2}=\pi R^{2} A \tau_{0}(1+\mu \cot \theta)+\pi R^{2} B \rho_{0} \sin ^{2} \theta(1+\mu \cot \theta) V_{z}^{2} z>p$

It can be written as follows:

$$
F_{z 2}=\alpha_{2}+\beta_{2} V_{z}^{2}
$$

where

$$
\begin{gathered}
\alpha_{2}=\pi R^{2} A \tau_{0}(1+\mu \cot \theta), \\
\beta_{2}=\pi R^{2} B \rho_{0} \sin ^{2} \theta(1+\mu \cot \theta) .
\end{gathered}
$$

According to the Newton second law, we can get

$$
m V_{z} \frac{d V_{z}}{d z}=-\left(\alpha_{2}+\beta_{2} V_{z}^{2}\right)
$$

Obtaining

$$
\frac{m}{2} \frac{d V_{z}^{2}}{\left(\alpha_{2}+\beta_{2} V_{z}^{2}\right)}=-d z
$$

Integrating

$$
-\frac{m}{2 \beta_{2}} \ln \left(\alpha_{2}+\beta_{2} V_{z}^{2}\right)+C_{2}=z
$$

When $z=p, V_{z}=V_{z p}$, getting

$$
C_{2}=\frac{m}{2 \beta_{2}} \ln \left(\alpha_{2}+\beta_{2} V_{z p}^{2}\right)+p
$$

Substituting $C_{2}$ into equation (26), we can get

$$
z=\frac{m}{2 \beta_{2}} \ln \left(\frac{\alpha_{2}+\beta_{2} V_{z p}^{2}}{\alpha_{2}+\beta_{2} V_{z}^{2}}\right)+p .
$$

Setting $V_{z}=0$, we can get the maximum penetrating depth $h_{2}\left(h_{2}>p\right)$.

$$
h_{2}=\frac{m}{2 \beta_{2}} \ln \left(1+\frac{\beta_{2}}{\alpha_{2}} V_{z p}^{2}\right)+p .
$$

By the way, according to the Newton second law, we can get

$$
m \frac{d V_{z}}{d t}=-\left(\alpha_{2}+\beta_{2} V_{z}^{2}\right)
$$

Integrating the above equation, we can get

$$
V_{z}=\sqrt{\frac{\alpha_{2}}{\beta_{2}}} \tan \left[-\frac{\sqrt{\alpha_{2} \beta_{2}}}{m} t+C_{3}\right] .
$$

Taking the initial condition $V_{z}\left(t_{p}\right)=V_{z p}\left(t_{p}\right.$ is the time of $z=p$ ) into the above equation, we can obtain the relationship between $V_{z}$ and the time $t$. Then, the relationship between the acceleration and the time can be known.

3.3. Penetrating Test. In order to verify the abovementioned penetration equation, the relationship between the penetrating depth and the penetrating speed of the anchor body in 


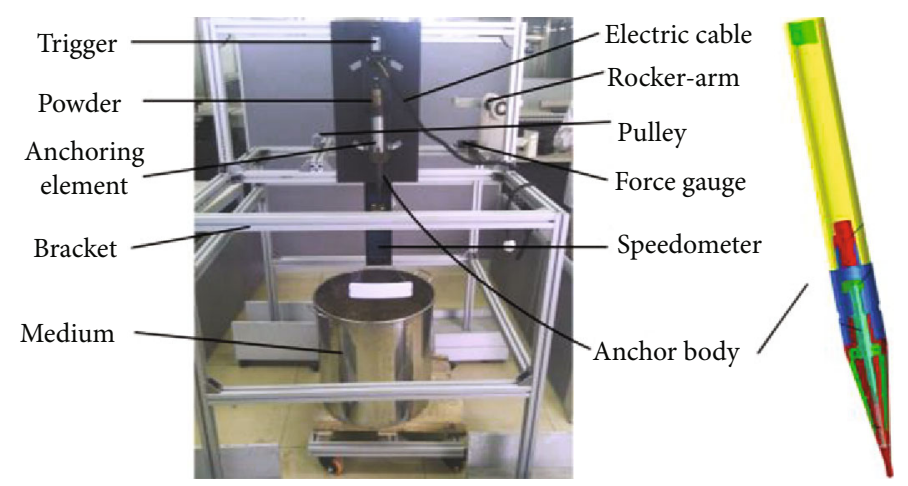

FIgURE 4: Testing platform of the anchoring system.

TABle 1: Parameters of the clay and fine sand.

\begin{tabular}{lcccccccc}
\hline $\begin{array}{l}\text { Parameter } \\
\text { Medium }\end{array}$ & $\begin{array}{c}\text { Density } \rho_{0} \\
\left(\mathrm{~kg} / \mathrm{m}^{3}\right)\end{array}$ & $\begin{array}{c}\text { Internal frictional } \\
\text { angle } \varphi\left({ }^{\circ}\right)\end{array}$ & $\begin{array}{c}\text { Cohesion } \tau_{0} \\
(\mathrm{kPa})\end{array}$ & $\begin{array}{c}\text { Elasticity module } E \\
(\mathrm{MPa})\end{array}$ & $\begin{array}{c}\text { Poisson } \\
\text { ratio }\end{array}$ & $\begin{array}{c}\text { Water } \\
\text { content }\end{array}$ & $\begin{array}{c}\text { Void } \\
\text { ratio }\end{array}$ & $\begin{array}{c}\text { Locked volumetric } \\
\text { strain } \eta *\end{array}$ \\
\hline Hard clay & $1.98 \times 10^{3}$ & 14 & 160 & 23.8 & 0.3 & $19.91 \%$ & 0.709 \\
Fine sand & $1.71 \times 10^{3}$ & 35.5 & 0.2 & 45.0 & 0.35 & 0 & 0.585 & 0.15 \\
Soft clay & $1.85 \times 10^{3}$ & 14 & 5.0 & - & - & - & - & - \\
\hline
\end{tabular}

hard clay and fine sand media is tested experimentally. Testing platform of the anchoring system is shown in Figure 4. The anchoring element is fired by the powder, which is ignited via the trigger, and the trigger is controlled by the electric cable. When the anchor body is fired by the anchoring element, the firing speed of the anchor body is measured by the speedometer, and the penetrating depth is measured by the thread on the rear of the anchor body. Then, the anchor body is pulled out of the medium by the rocker-arm via the pulley. There is a force gauge between the rockerarm and the pulley; thus, the anchoring force of the anchoring element will be measured. The diameter of anchor body (penetrating unit) used in the test is about $20 \mathrm{~mm}$, and the mass is about $160 \mathrm{~g}$.

The parameters of the media are shown in Table 1. Hard clay and fine sand are used in penetrating tests, and soft clay is used in anchoring tests in Section 4.2. Figure 5 shows the prediction of the penetration equation and the corresponding experimental results. It can be seen that the penetration equation can logically describe the correspondence between the penetrating depth and the penetrating speed. As the depth of the experimental media is about $0.5 \mathrm{~m}$, the experiment cannot penetrate the depth that exceeds $0.5 \mathrm{~m}$. Experimental result errors are mainly from the media parameters error and equipment measurement error. Simultaneously, it can be found that the depth increase in fine sand is less than that in hard clay as the penetrating speed increases. This phenomenon is related to the fluidity (characterized by the cohesion) of the fine sand. The smaller the cohesion, the easier it is to flow. Thus, the cavity formed in fine sand as the anchor body penetrating is difficult to maintain. Compared with hard clay, flowing fine sand is easier to consume the penetration energy of the anchor body.

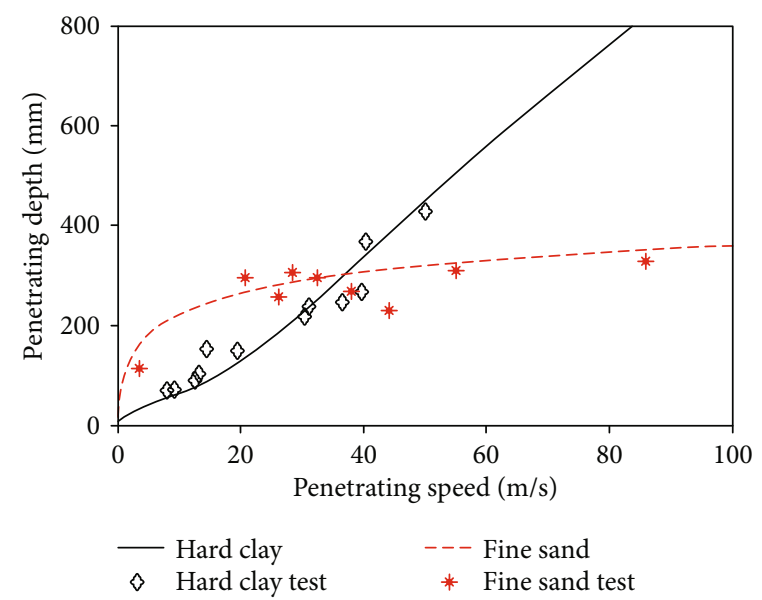

Figure 5: Results of penetration equation and experiments of anchor penetration.

\section{Anchoring Model}

4.1. Anchoring Force Analysis. After the anchor body has penetrated, the barbs on the anchor tip are opened to produce a greater anchoring force. The shape of the open barbs formed can be approximately regarded to be round and like a disc. There will be shear damage between the round disc anchor tip and the medium. It is found that a circular truncated cone concave was formed on the surface of the medium after the anchor body is pulled out. Thus, an anchoring model shown in Figure 6 is established. The maximum principal stress of the element in medium is $\sigma_{1}$, which is the passive stress of the Rankine. The minimum principal stress $\sigma_{3}$ is the compressive stress caused by the gravity of the upper layer. The depth of the round disc on the anchor tip is $H$. 


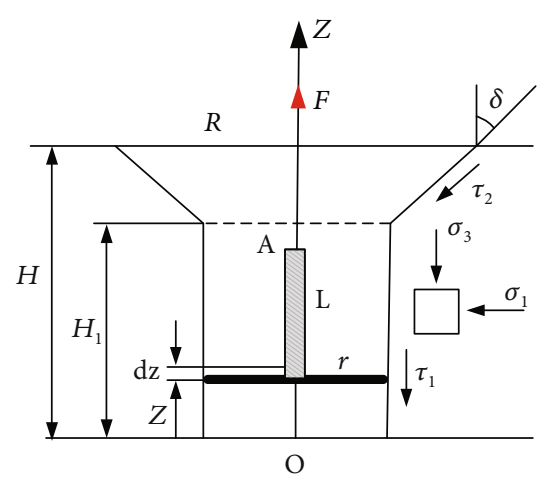

FIGURE 6: Anchoring model of the anchoring system after barbs stretching.

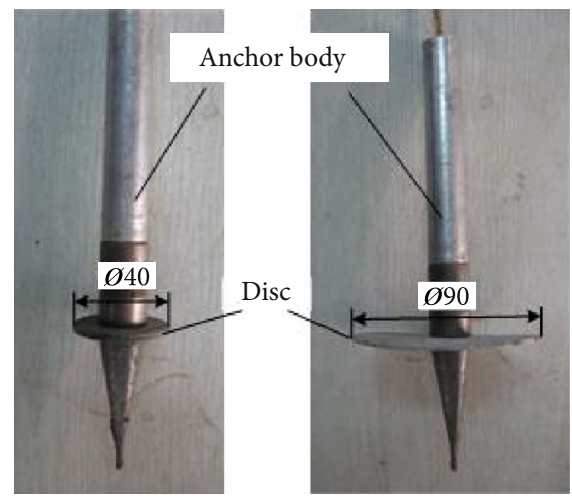

Figure 7: $40 \mathrm{~mm}$ and $90 \mathrm{~mm}$ diameter disc mounted on the anchor body.

The medium is sheared layedly when the anchor is pulled out. When the round disc of the anchor tip is pulled out by the distance of $H_{1}$ and reaches A position, the anchoring force $F$ reaches the maximum value. Then, under the effect of this maximum force, the surface of the medium is subjected to the overall shear failure and forms the circular truncated cone concave. The height of the circular truncated cone concave is $H-H_{1}$. Its bottom radius is $R$, and its top radius is equal to the round disc radius on the anchor tip, which is $r$. The angle between medium surface normal and the concave generatrix is $\delta=\pi / 4+\varphi / 2$ (based on soil mechanics, the angle between the shear failure surface and the maximum principal stress surface is $\delta=\pi / 4+\varphi / 2$ ). Finally, the anchor tip is separated from the medium by holding the circular truncated cone.

The maximum principal stress $\sigma_{1}$ and the minimum principal stress $\sigma_{3}$ are

$$
\begin{gathered}
\sigma_{1}=\rho g(H-z) \tan ^{2} \delta+2 \tau_{0} \tan \delta, \\
\sigma_{3}=\rho g(H-z),
\end{gathered}
$$

where $\rho$ is the density of the medium and $g$ is the gravity coefficient.

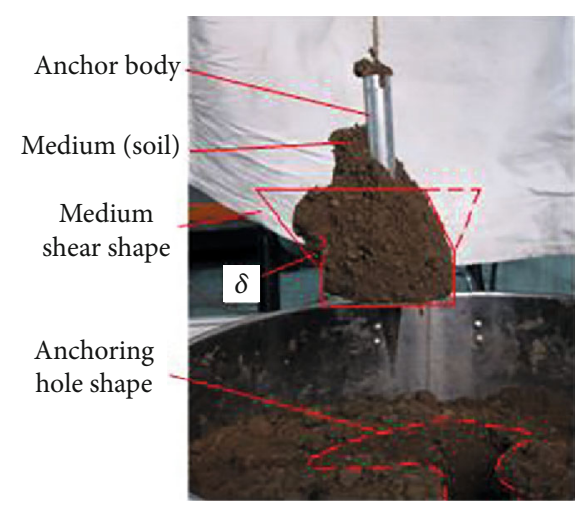

FIgURE 8: Medium (soil) and its shape when the anchor body is pulled out.

From the Mohr-Coulomb yield criterion, the shear strength of the medium is

$$
\tau=\tau_{0}+\sigma \tan \varphi .
$$

The positive pressure $\sigma$ of the medium in column shear failure stage $\left(0 \leq z \leq H_{1}\right)$ and circular truncated cone shear failure stage $\left(H_{1}<z \leq H\right)$, respectively, are

$$
\sigma= \begin{cases}\sigma_{1} & 0 \leq z \leq H_{1}, \\ \frac{\sigma_{1}+\sigma_{3}}{2}+\frac{\sigma_{1}-\sigma_{3}}{2} \cos 2 \delta & H_{1}<z \leq H .\end{cases}
$$

Thus, the shear strength is as follows:

$$
\begin{array}{cc}
\tau_{1}=\tau_{0}+\sigma_{1} \tan \varphi & 0 \leq z \leq H_{1}, \\
\tau_{2}=\tau_{0}+\left(\frac{\sigma_{1}+\sigma_{3}}{2}-\frac{\sigma_{1}-\sigma_{3}}{2} \sin \varphi\right) \tan \varphi & H_{1}<z \leq H .
\end{array}
$$

The anchoring force $F_{1}$ generated in column shear failure stage $\left(0 \leq z \leq H_{1}\right)$ can be expressed as follows:

$$
F_{1}=\int_{0}^{z} 2 \pi r \tau_{1} d z
$$

The anchoring force $F_{2}$ generated in circular truncated cone shear failure stage $\left(H_{1}<z \leq H\right)$ can be expressed as follows:

$$
F_{2}=\int_{H_{1}}^{H} 2 \pi\left[r+\left(z-H_{1}\right) \tan \delta\right] \tau_{2} \frac{d z}{\cos \delta} .
$$

According to the assumption of the anchoring model, when $z=H_{1}$, the anchoring force reaches the maximum value, and this force causes the shear failure of part $\left(H-H_{1}\right)$, resulting in a circular truncated cone concave. Thus,

$$
F_{1}\left(z=H_{1}\right)=F_{2} \cos \delta
$$




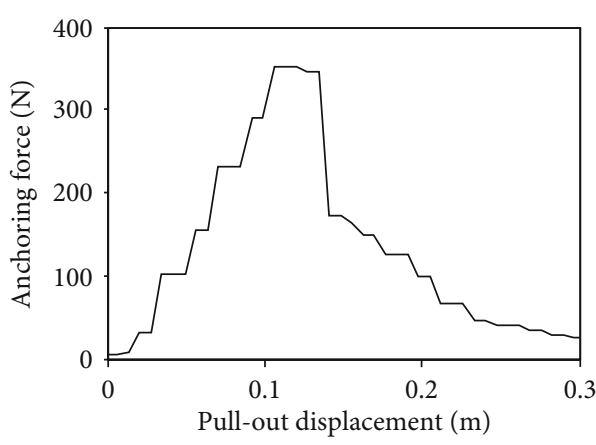

(a)

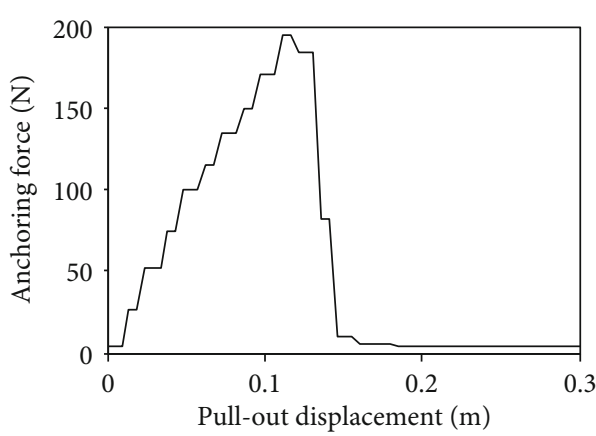

(c)

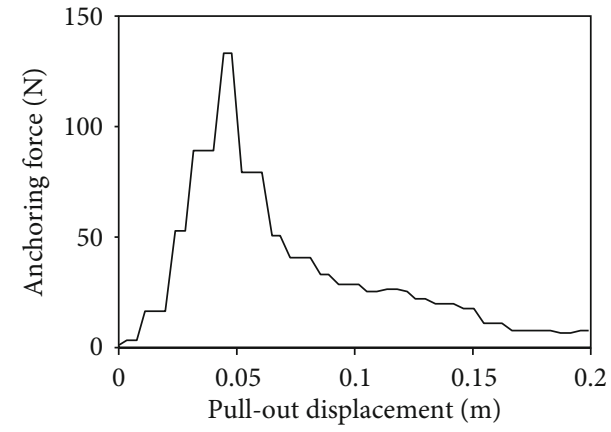

(b)

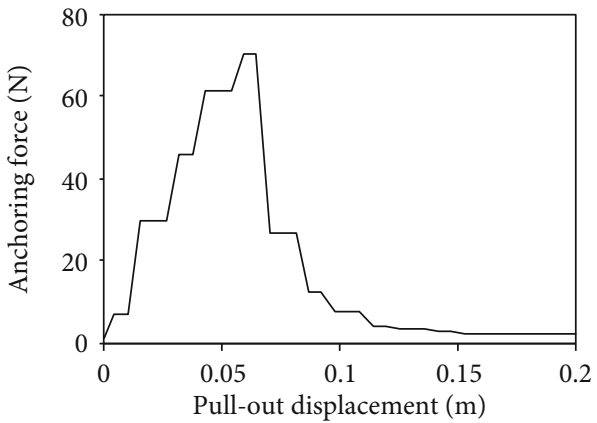

(d)

FIgURE 9: Anchoring experiments in soft clay.

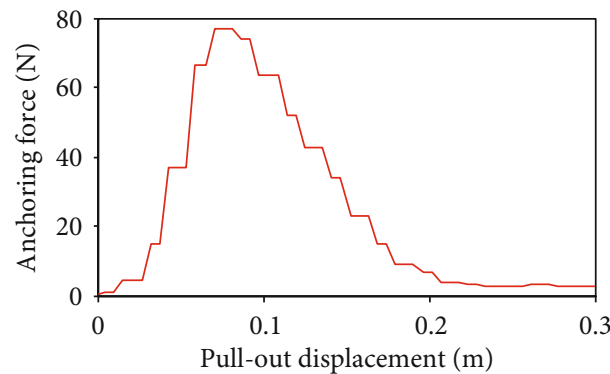

(a)

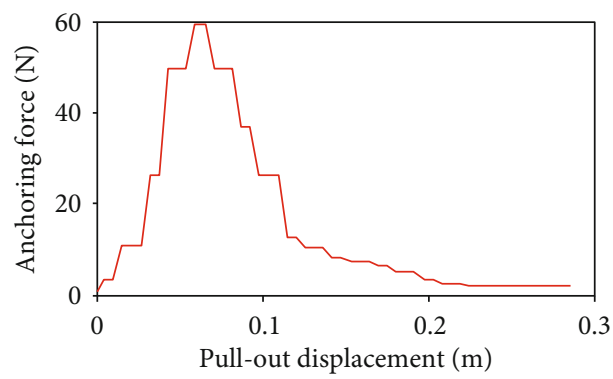

(c)

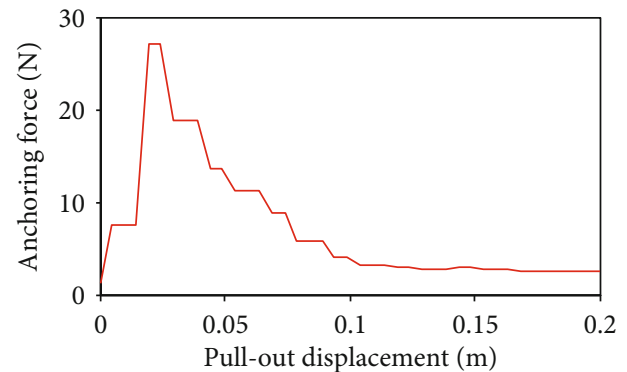

(b)

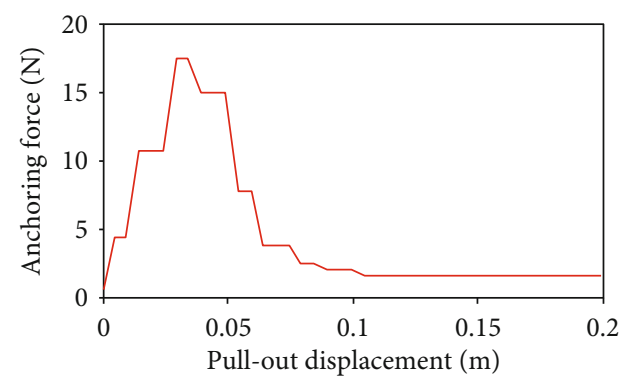

(d)

FIgURE 10: Anchoring experiments in fine sand (also called soft sand).

Jointing equations (30) and (33) (36), we can firstly obtain $H_{1}$. Then, the value of the anchoring force in $0 \leq z \leq$ $H_{1}$ interval can be obtained.

When the circular truncated cone is generated after shear failure of the medium $\left(z>H_{1}\right)$, the anchoring force $F_{3}$ is the gravity of the circular truncated cone pulled out by the anchor tip. It can be expressed as follows:

$$
F_{3}=\int_{H_{1}}^{H} \rho g \pi\left[r+\left(z-H_{1}\right) \tan \delta\right]^{2} d z .
$$


TABLE 2: Comparison of anchoring model with experiments in soft clay and sand.

\begin{tabular}{|c|c|c|c|c|c|}
\hline & & $\begin{array}{c}H=285 \mathrm{~mm} \\
2 r=90 \mathrm{~mm}\end{array}$ & $\begin{array}{c}H=165 \mathrm{~mm} \\
2 r=90 \mathrm{~mm}\end{array}$ & $\begin{array}{c}H=285 \mathrm{~mm} \\
2 r=40 \mathrm{~mm}\end{array}$ & $\begin{array}{c}H=165 \mathrm{~mm} \\
2 r=40 \mathrm{~mm}\end{array}$ \\
\hline \multirow{2}{*}{ Soft clay } & Maximum anchoring force in the anchoring model & $348.8 \mathrm{~N}$ & $131.5 \mathrm{~N}$ & $176.1 \mathrm{~N}$ & $67.0 \mathrm{~N}$ \\
\hline & Maximum anchoring force in the tests & $355.0 \mathrm{~N}$ & $133.6 \mathrm{~N}$ & $195.4 \mathrm{~N}$ & $70.1 \mathrm{~N}$ \\
\hline \multirow{2}{*}{ Fine sand } & Maximum anchoring force in the anchoring model & $84.8 \mathrm{~N}$ & $20.9 \mathrm{~N}$ & $61.0 \mathrm{~N}$ & $13.7 \mathrm{~N}$ \\
\hline & Maximum anchoring force in the tests & $77.0 \mathrm{~N}$ & $27.1 \mathrm{~N}$ & $59.3 \mathrm{~N}$ & $17.5 \mathrm{~N}$ \\
\hline
\end{tabular}

Thus, the anchoring force $F$ in the whole pulling out process of the anchor body can be expressed as follows:

$$
F= \begin{cases}F_{1} & 0 \leq z \leq H_{1} \\ F_{3} & z>H_{1} .\end{cases}
$$

4.2. Anchoring Test. The anchoring model is verified by testing the anchoring forces at different depths in fine sand and soft clay media. After several times of penetratings, the state of the hard clay is destroyed and its parameters are changed. We remeasure the parameters of the clay and call the clay in this state as soft clay. Moreover, soft clay is convenient for operating in test. The key parameters of the fine sand and the soft clay are shown in Table 1 . Discs with $90 \mathrm{~mm}$ diameter and $40 \mathrm{~mm}$ diameter are separately mounted on the top of the anchor body, which is shown in Figure 7. Then, the anchoring forces of the anchor body in two different penetrating depths are tested. There are two penetrating depth values: one is $285 \mathrm{~mm}$ which represents full penetrating of the anchor body, and the other is $165 \mathrm{~mm}$ representing partial penetrating.

The medium on the anchor body after it is pulled out from the soft soil in $285 \mathrm{~mm}$ penetrating depth is shown in Figure 8, where the anchoring hole shape is also displayed. As the medium on the anchor body would collapse under the effect of the gravity, it is difficult to find the integral shear shape of the medium on the anchor body at the time of pulling out. Nonetheless, it is obvious that the medium shear shape on the left side of the anchor body in Figure 8 is very similar to that shown in Figure 6.

When the anchor body is pulled out from the soft clay and the fine sand, the relationship between the anchoring force and the pullout displacement is shown in Figures 9 and 10, and the maximum anchoring force can be found in two figures. We can conclude from the testing that the anchoring force increases gradually during the pullout process, and the surface of the media is ruptured suddenly when the anchoring force is up to the maximum (due to strong liquidity of the sand, the phenomenon is not obvious). After the anchoring force decreases to a constant value, this value equals to the total weight of the anchor body, the disc, and the medium on its surface. At the same time, it can be seen that the cohesion of the medium, the internal friction angle, the penetration depth of the anchor body, and the diameter of the disc have great influence on the anchoring force.

The calculated maximum anchoring force can be obtained by substituting the parameters of the experimental medium and the penetrating depth of the anchor body into the anchoring model. The calculated and the testing maximum anchoring force are shown in Table 2. It can be found that the calculated maximum anchoring force is close to the maximum anchoring force obtained by the experiment, so the anchoring model is considered as reasonable.

\section{Penetrating-Anchoring Mathematical Model}

At the time of the asteroid landing mechanism landing, the anchor body of the anchoring system is projected into the asteroid surface at a certain speed, which produces the anchoring force. The anchoring force is the key performance index for the asteroid landing mechanism. Establishing the relationship between the initial penetrating speed of the anchor body and the anchoring force is of great importance.

The anchoring model mainly studies the relationship between the penetrating depth $H$ and the anchoring force $F$ , and the penetration equation mainly describes the relationship between the penetration depth $H$ and the initial penetrating velocity $V_{0}$. Therefore, on the base of the anchoring model and the penetration equation, the mathematical relationship between the initial penetrating velocity $V_{0}$ and the anchoring force $F$ can be established with the penetration depth $H$ as the link. Through this mathematical relationship, we can easily calculate the minimum initial penetrating speed needed to obtain the required anchoring force.

The penetrating depth of the anchor body can be obtained by the penetrating equations (16) and (27), which can be expressed as follows:

$$
H= \begin{cases}h_{1} & 0 \leq z \leq p, \\ h_{2} & z>p\end{cases}
$$

written as

$$
H=f_{1}\left(m, V_{0}, \theta, \mu, \tau_{0}, E, \varphi, \rho, \rho *\right)
$$

When the anchor body is anchored at the depth $H$, the maximum anchoring force occurrence point $H_{1}$ can be obtained by combining equations (30) and (33) (36). It is written as follows:

$$
H_{1}=f_{2}\left(H, \tau_{0}, \varphi, \rho, g\right)
$$

And then substituting the $z=H_{1}$ into equation (34), we can obtain the maximum anchoring force $F_{\max }$.

$$
F_{\text {max }}=f_{3}\left(H, \tau_{0}, \varphi, \rho, g\right) \text {. }
$$


Substituting equation (40) into equation (42), we obtain

$$
F_{\max }=f_{4}\left(m, V_{0}, \theta, \mu, \tau_{0}, E, \varphi, \rho, \rho *, g\right) .
$$

Equation (43) is the penetrating-anchoring model. This model incorporates the penetration equation and the anchoring model with the penetrating depth as a link, which combines two independent processes (penetrating and anchoring) as a whole. This model describes the mathematical relationship among the maximum anchoring force $F_{\text {max }}$, the initial penetrating velocity $V_{0}$, and the medium properties. The penetrating-anchoring model can easily predict the required minimum initial penetrating speed that is adopted for generating the expected maximum anchoring force.

\section{Conclusions}

In the paper, based on the cavity expansion model and the Mohr-Coulomb yield criterion, the force on the anchor body during the penetration is analyzed. A penetrating equation is introduced for calculating the resistance force and penetrating depth of the anchor body when it is penetrating. The penetrating depth estimated by the model is approximate to the testing results in clay and sand media. Utilizing soil mechanics, the force on the deployed anchor body in the process of pulling out is analyzed, and the anchoring model is built. This model describes the relationship between the penetrating depth and the anchoring force. The anchoring force on the anchor body equipped with $40 \mathrm{~mm}$ disc and the $90 \mathrm{~mm}$ disc which represent deployed anchor tip are tested in the clay and fine sand. The maximum anchoring force obtained by the tests is close to the calculated maximum anchoring force. The maximum anchoring force in the test appears at the time of media surface failure, and the residual medium's shape on the anchor body after it is extracted is similar to the medium's shear shape in the anchoring model. The penetrating-anchoring model is built by uniting the anchoring model and the penetration equation with the penetration depth as the link. This model establishes the relationship among the anchoring force, the initial penetrating velocity, and the media properties, and it provides a theoretical foundation for the design and performance estimation of the anchoring system in the asteroid or the comet landing exploration. Tests from different angles or close to the actual situation are the follow-up plan, and we are planning to carry out similar tests in the future.

\section{Data Availability}

The data used to support the findings of this study are available from the corresponding author upon request.

\section{Conflicts of Interest}

The authors declare that they have no conflicts of interest.

\section{Acknowledgments}

This work is financially supported by the National Natural Science Foundation of China (No. 51775129 and No. 51975139) and the State Key Laboratory of Robotics and System (HIT).

\section{References}

[1] R. P. Binzel, D. F. Lupishko, M. D. Martino, R. J. Whiteley, and G. J. Hahn, "Physical properties of near-earth objects," in Asteroids III, pp. 255-271, University of Arizona Press, 2002.

[2] B. R. Blair, The role of near-Earth asteroids in long-term platinum supply, Lunar and Planetary Institute, Golden, Colorado, 2000.

[3] E. Gibney, "Mission accomplished: Rosetta crashes into comet," Nature News, vol. 538, no. 7623, pp. 13-14, 2016.

[4] W. B. Xu and H. B. Zhao, "Deep space exploration of asteroids: the science perspectives," Advance in Earth Sciences, vol. 20, no. 11, pp. 1183-1190, 2005.

[5] S. Ulamec, C. Fantinati, M. Maibaum et al., "Rosetta landerlanding and operations on comet 67P/Churyumov Gerasimenko," Acta Astronautica, vol. 125, pp. 80-91, 2016.

[6] K. Ilamparuthi and E. A. Dickin, "The influence of soil reinforcement on the uplift behaviour of belled piles embedded in sand," Geotextiles and Geomembranes, vol. 19, no. 1, pp. 1-22, 2001.

[7] H. B. Sutherland, "Uplift resistance of soils," Geotechnique, vol. 38, no. 4, pp. 493-516, 1988.

[8] E. A. Dickin, "Uplift behavior of horizontal anchor plates in sand," Journal of Geotechnical Engineering, vol. 114, no. 11, pp. 1300-1317, 1988.

[9] K. S. Rao and J. Kumar, "Vertical uplift capacity of horizontal anchors," Journal of Geotechnical Engineering, vol. 120, no. 7, pp. 1134-1147, 1994.

[10] R. S. Merifield, A. V. Lyamin, S. W. Sloan, and H. S. Yu, "Three-dimensional lower bound solutions for stability of plate anchors in clay," Journal of Geotechnical and Geoenvironmental Engineering, vol. 129, no. 3, pp. 243-253, 2003.

[11] Z. M. Wu, S. T. Yang, X. Z. Hu, and J. Zheng, “Analytical method for pullout of anchor from anchor-mortar-concrete anchorage system due to shear failure of mortar," Journal of Engineering Mechanics, vol. 133, no. 12, pp. 1352-1369, 2007.

[12] K. Shanker, P. K. Basudhar, and N. R. Patra, "Uplift capacity of single piles: predictions and performance," Geotechnical and Geological Engineering, vol. 25, no. 2, pp. 151-161, 2007.

[13] H. Niroumand, K. A. Kassim, A. Ghafooripour, and R. Nazir, "Uplift capacity of enlarged base piles in sand," Electronic Journal of Geotechnical Engineering, vol. 17, pp. 2721-2737, 2012.

[14] J. Liu, M. Liu, and Z. Zhu, "Sand deformation around an uplift plate anchor," Journal of Geotechnical and Geoenvironmental Engineering, vol. 138, no. 6, pp. 728-737, 2012.

[15] N. C. Consoli, C. A. Ruver, and F. Schnaid, "Uplift performance of anchor plates embedded in cement-stabilized backfill," Journal of Geotechnical and Geoenvironmental Engineering, vol. 139, no. 3, pp. 511-517, 2013.

[16] J. B. Xu, Investigations on Long Projectiles Penetrating into Concrete Targets, National University of Defense Technology (Doctoral dissertation), 2001. 
[17] T. He, A Study on the Penetration of Projectiles into Targets Made of Various Materials, University of Science and Technology of China (Doctoral dissertation), 2007.

[18] M. J. Forrestal and V. K. Luk, "Penetration into soil targets," International Journal of Impact Engineering, vol. 12, no. 3, pp. 427-444, 1992.

[19] Z. Zhao, S. Wang, D. Li, H. Wang, Y. Wang, and J. Zhao, "Development of an anchoring system for the soft asteroid landing exploration," International Journal of Aerospace Engineering, vol. 2019, Article ID 1257038, 13 pages, 2019. 